\title{
Preliminary Screening for Hereditary Breast and Ovarian Cancer Using a Chatbot Augmented Intelligence Genetic Counselor: Development and Feasibility Study
}

Ann Sato ${ }^{1}$, MSc; Eri Haneda ${ }^{1}, \mathrm{MSc}$; Nobuyasu Suganuma ${ }^{2}, \mathrm{MD}, \mathrm{PhD}$; Hiroto Narimatsu ${ }^{1,3,4}, \mathrm{MD}, \mathrm{PhD}$

\footnotetext{
${ }^{1}$ Department of Genetic Medicine, Kanagawa Cancer Center, Yokohama, Kanagawa, Japan

${ }^{2}$ Department of Breast and Endocrine Surgery, Kanagawa Cancer Center, Yokohama, Kanagawa, Japan

${ }^{3}$ Cancer Prevention and Cancer Control Division, Kanagawa Cancer Center Research Institute, Yokohama, Kanagawa, Japan

${ }^{4}$ Graduate School of Health Innovation, Kanagawa University of Human Services, Kawasaki, Kanagawa, Japan
}

\section{Corresponding Author:}

Hiroto Narimatsu, MD, $\mathrm{PhD}$

Department of Genetic Medicine

Kanagawa Cancer Center

2-3-2 Nakao, Asahi-ku

Yokohama, Kanagawa, 241-8515

Japan

Phone: 810455202222

Email: hiroto-narimatsu@umin.org

\section{Abstract}

Background: Breast cancer is the most common form of cancer in Japan; genetic background and hereditary breast and ovarian cancer (HBOC) are implicated. The key to HBOC diagnosis involves screening to identify high-risk individuals. However, genetic medicine is still developing; thus, many patients who may potentially benefit from genetic medicine have not yet been identified.

Objective: This study's objective is to develop a chatbot system that uses augmented intelligence for HBOC screening to determine whether patients meet the National Comprehensive Cancer Network (NCCN) BRCA1/2 testing criteria.

Methods: The system was evaluated by a doctor specializing in genetic medicine and certified genetic counselors. We prepared 3 scenarios and created a conversation with the chatbot to reflect each one. Then we evaluated chatbot feasibility, the required time, the medical accuracy of conversations and family history, and the final result.

Results: The times required for the conversation were 7 minutes for scenario 1,15 minutes for scenario 2 , and 16 minutes for scenario 3. Scenarios 1 and 2 met the BRCA1/2 testing criteria, but scenario 3 did not, and this result was consistent with the findings of 3 experts who retrospectively reviewed conversations with the chatbot according to the 3 scenarios. A family history comparison ascertained by the chatbot with the actual scenarios revealed that each result was consistent with each scenario. From a genetic medicine perspective, no errors were noted by the 3 experts.

Conclusions: This study demonstrated that chatbot systems could be applied to preliminary genetic medicine screening for HBOC.

(JMIR Form Res 2021;5(2):e25184) doi: 10.2196/25184

\section{KEYWORDS}

artificial intelligence; augmented intelligence; hereditary cancer; familial cancer; IBM Watson; preliminary screening; cancer; genetics; chatbot; screening; feasibility

\section{Introduction}

Breast cancer is the most common form of cancer in Japan, with approximately 90,000 new cases every year. Approximately 10,000 patients in Japan die from breast cancer each year [1]. Approximately $5 \%-10 \%$ of breast cancers are strongly related to genetic background, and of these, hereditary breast and ovarian cancer (HBOC) is the most common [2-4]. HBOC is an autosomal-dominant disease that is diagnosed based on the presence of BRCA1 or BRCA2 (BRCA1/2) pathogenic germline mutations. The BRCA1/2 genes encode 2 proteins involved in DNA damage repair. Recent studies have shown that mutations 
in BRCA1/2 and several other genes, including TP53 and PALB2, can lead to hereditary breast cancer in Japanese women [5]. Reportedly, BRCA1 and BRCA2 mutation carriers have cumulative breast cancer risks of $72 \%$ and $69 \%$, respectively, and cumulative ovarian cancer risks of $44 \%$ and $17 \%$, respectively, up to the age of 80 years. These risks are remarkably higher than those in the general population [6]. Therefore, genetic counseling and testing are recommended for individuals with suspected $\mathrm{HBOC}$ and relatives of carriers of the BRCA1/2 mutation. For carriers of the BRCA1/2 mutation diagnosed by genetic testing, appropriate countermeasures such as surveillance or risk reduction surgery can be considered [7].

A diagnosis of HBOC relies on a screening procedure to identify those at a high risk of disease, and therefore, the collection of information about family history is very important. However, genetic medicine is still developing in Japan. Even in the United States, many patients who might potentially benefit from genetic medicine have not been identified. A previous study suggested that this gap may be caused by a lack of awareness and knowledge among health care workers and patients, delays in updating information due to frequent revisions of the BRCA1/2 testing criteria, and a lack of human resources and health care workers responsible for identifying these patients [8].

In Japan, the preliminary screening of patients at high risk of HBOC is usually conducted by a certified genetic counselor (CGC). High-risk patients who undergo screening are recommended to receive genetic counseling to enable them to decide whether to undergo genetic testing. As of April 2020, however, there were only 267 CGCs in Japan [9], and it is difficult to rapidly increase this number because advanced specialized education is provided at a limited number of facilities.

In recent years, we have focused on developing augmented intelligence, which has been applied for practical use. Augmented intelligence has several functions, including a chatbot mechanism, which is software that allows users to interact with the system through an algorithm without the need for human back-end intervention. Chatbots have been applied in previous research as a communication support tool for patients with cancer [10-12]. Reportedly, chatbots improved medication adherence in patients with breast cancer and were able to provide support and anxiety reduction in young adults who underwent cancer treatment. The chatbot was useful for both younger patients with cancer as well as for the health monitoring of older patients ( $\geq 65$ years) with cancer. These studies suggested that chatbots are useful in the medical field, especially in supporting patients with cancer.

There are many advantages of using chatbots for preliminary screening in medical practices. First, the chatbot can independently conduct preliminary screening automatically and could thus handle some of the CGC's routine work. The CGC would then be able to focus on more intricate work, such as personalized genetic counseling, which would improve productivity. Second, a chatbot for preliminary screening is easy to create because the purpose of the conversation is clear, and the flow of information collection used for determining whether the BRCA1/2 testing criteria are met has a pattern. Lastly, the chatbot will enable the identification of a larger number of patients; traditional preliminary screening requires time and effort, which could be resolved with the chatbot.

This study aims to develop a system based on IBM Watson (the chatbot system developed by IBM Corp) that would inquire about patients' family and medical histories and would identify which patients with breast cancer should be contacted by a CGC. The friendly interface created using augmented intelligence would be easy for the patients to understand. The study endpoint involves an evaluation of the developed system's clinical feasibility, which would ideally increase the number of targeted people who are identified by the preliminary screening and thus enable the provision of genetic medicine to a larger number of people in the future.

\section{Methods}

\section{Chatbot System}

This is a feasibility study by simulation using a scenario. The study's endpoint is to assess the accuracy of the family history heard by the chatbot system. Furthermore, as a preclinical study, we listened to the medical and family history of the actual medical staff and evaluated its accuracy.

We developed a system using the chatbot function of Watson, a service of IBM Corp's cognitive computing system. In this study, we used the real-time conversational interface LINE, a social network service (SNS) provided by LINE Corporation. LINE is the most popular SNS in Japan, and we assumed that it would be familiar to the patients [13]. Using this interface, we established a system that targeted patients with breast cancer who visited a designated hospital and assessed whether they met the National Comprehensive Cancer Network (NCCN) guidelines for BRCA1/2 testing criteria, version 3.2019, using information given in the patients' replies. The patients' responses were stored in the terminal (ie, a smartphone or tablet). Finally, the medical history, the family history, and the final results of preliminary screening appeared on the terminal screen. The development of this system was supported by the Advanced Integration Technology (AIT) Corporation. We set the genetic counselor, named "AI," as the persona to ensure that the patients could type to and interact with a familiar entity. Textbox 1 shows the persona of AI.

This study was approved by the institutional review board for the research of Kanagawa Cancer Center (2018 - epidemiology $55)$. 
Textbox 1. Summary of the characteristics of the chatbot genetic counselor, AI.

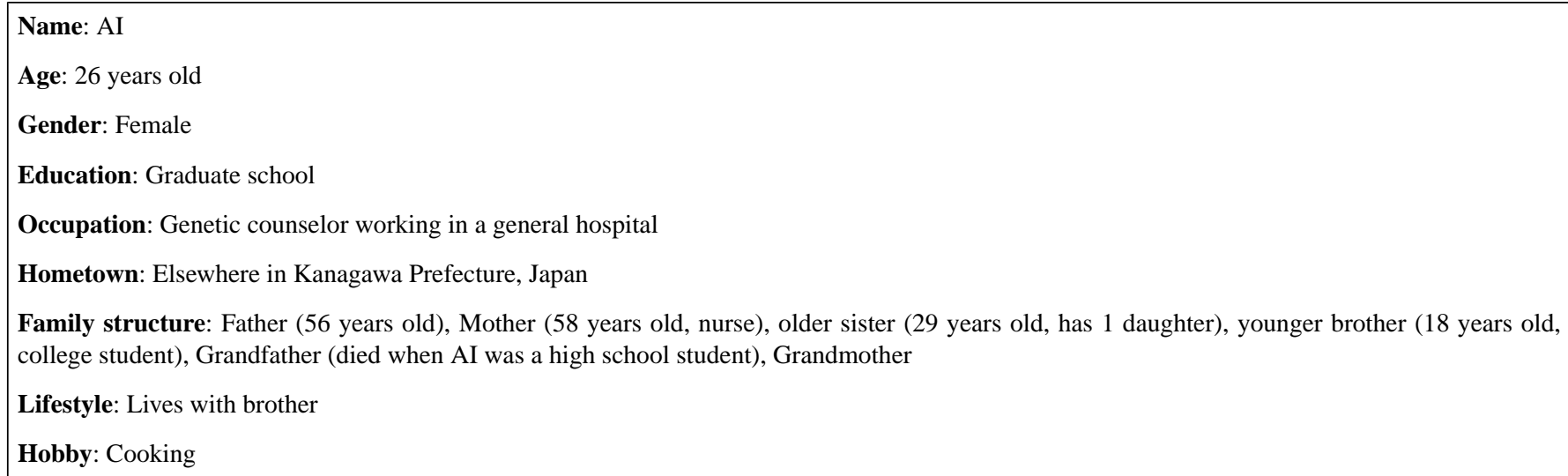

\section{Modification of the Guideline}

In this study, we partially modified the BRCA1/2 testing criteria according to our clinical practice at Kanagawa Cancer Center. The following items were omitted because the chatbot was not able to ask these questions of the patients: (1) individual from a family with a known BRCA1/2 pathogenic/likely pathogenic variant, including such variants found on research testing; (2) personal history of breast cancer diagnosed at 41-50 years old with $\geq 1$ close blood relative with high-grade (Gleason score $\geq 7$ ) prostate cancer; (3) personal history of breast cancer diagnosed at $\leq 60$ years old with triple-negative breast cancer; (4) personal history of breast cancer diagnosed at any age with $\geq 1$ close blood relative with metastatic prostate cancer or $\geq 2$ additional diagnoses of breast cancer at any age in close blood relatives; (5) personal history of metastatic prostate cancer; (6) personal history of high-grade prostate cancer (Gleason score $\geq 7$ ) at any age with $\geq 1$ close blood relative with ovarian carcinoma, pancreatic cancer, or metastatic prostate cancer at any age or breast cancer $<50$ years old, or $\geq 2$ close blood relatives with breast or prostate cancer (any grade) at any age; (7) BRCA1/2 pathogenic/likely pathogenic variant detected by tumor profiling on any tumor type in the absence of germline pathogenic/likely pathogenic variant analysis; (8) regardless of family history, some individuals with BRCA-related cancer who may benefit from genetic testing to determine targeted treatment eligibility.

The following item was omitted because it is rarely encountered in clinical practice in Japan: (1) personal history of breast cancer with Ashkenazi Jewish ancestry; (2) personal history of high-grade prostate cancer (Gleason score $\geq 7$ ) at any age with Ashkenazi Jewish ancestry.

The final results were presented to demonstrate whether the patient met these modified criteria.

\section{Evaluation of Feasibility}

System development and evaluation were conducted by a doctor specializing in genetic medicine (author HN) and 2 CGCs (authors AS and EH). We prepared 3 scenarios with 3 pedigrees (Figures 1-3) and created a conversation with the chatbot along the lines of these scenarios. Then, we evaluated chatbot feasibility, the required time, the medical accuracy of the conversation and the family history, and the final result.

To test the system, 3 experts used this system based on their family histories and evaluated the accuracy of the family histories and the final results. 
Figure 1. The family tree in scenario 1.

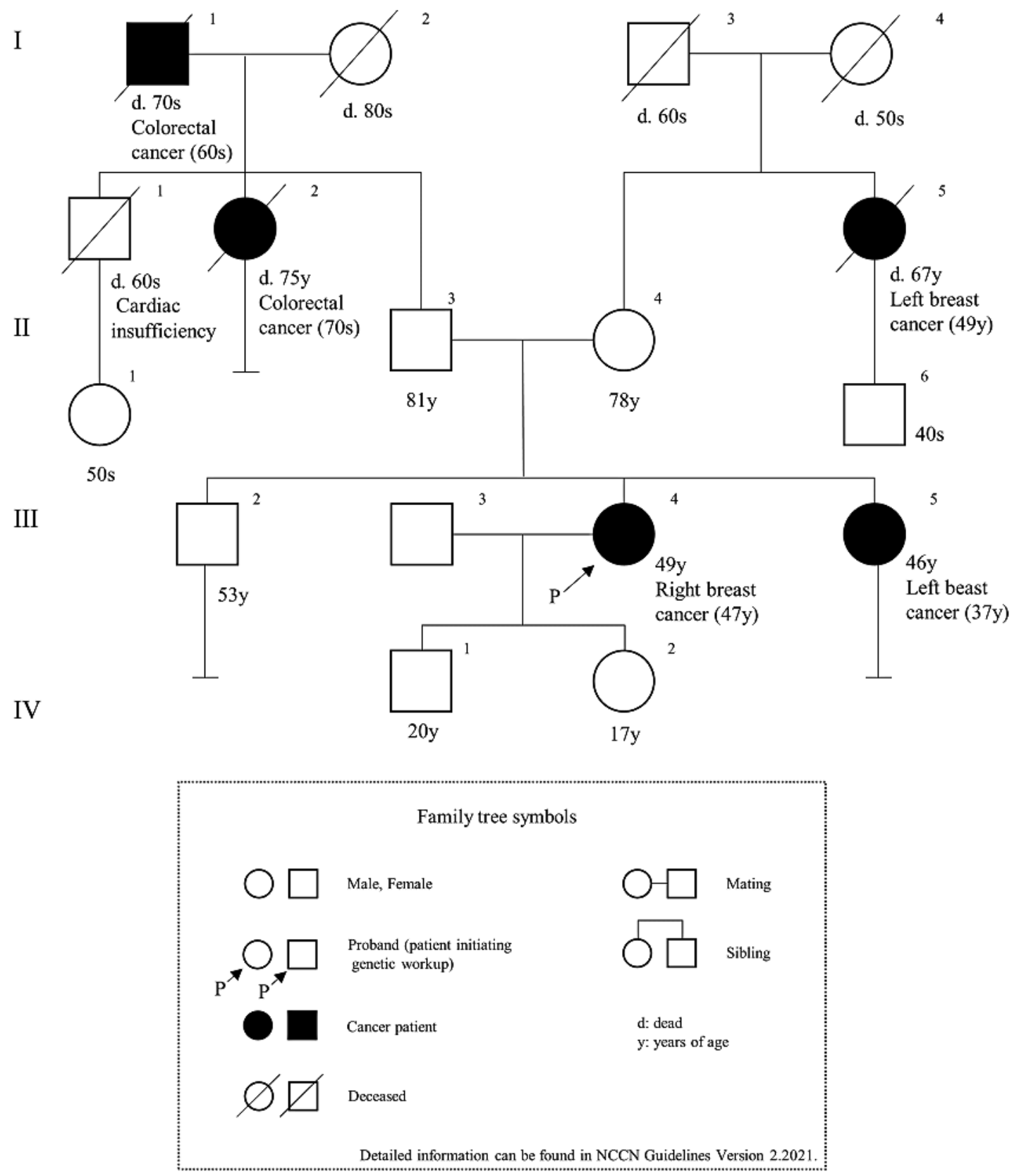


Figure 2. The family tree in scenario 2 .
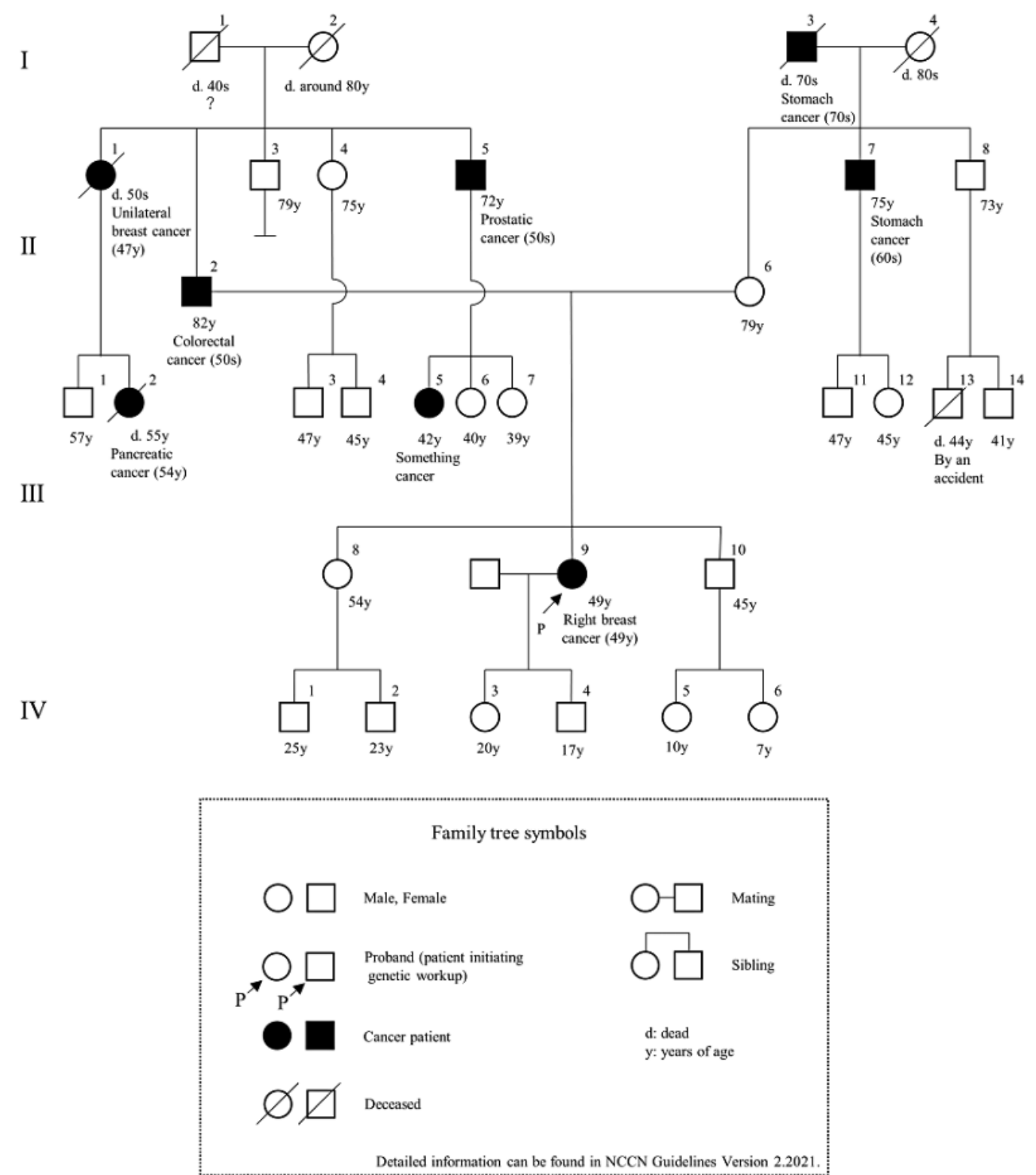
Figure 3. The family tree in scenario 3.

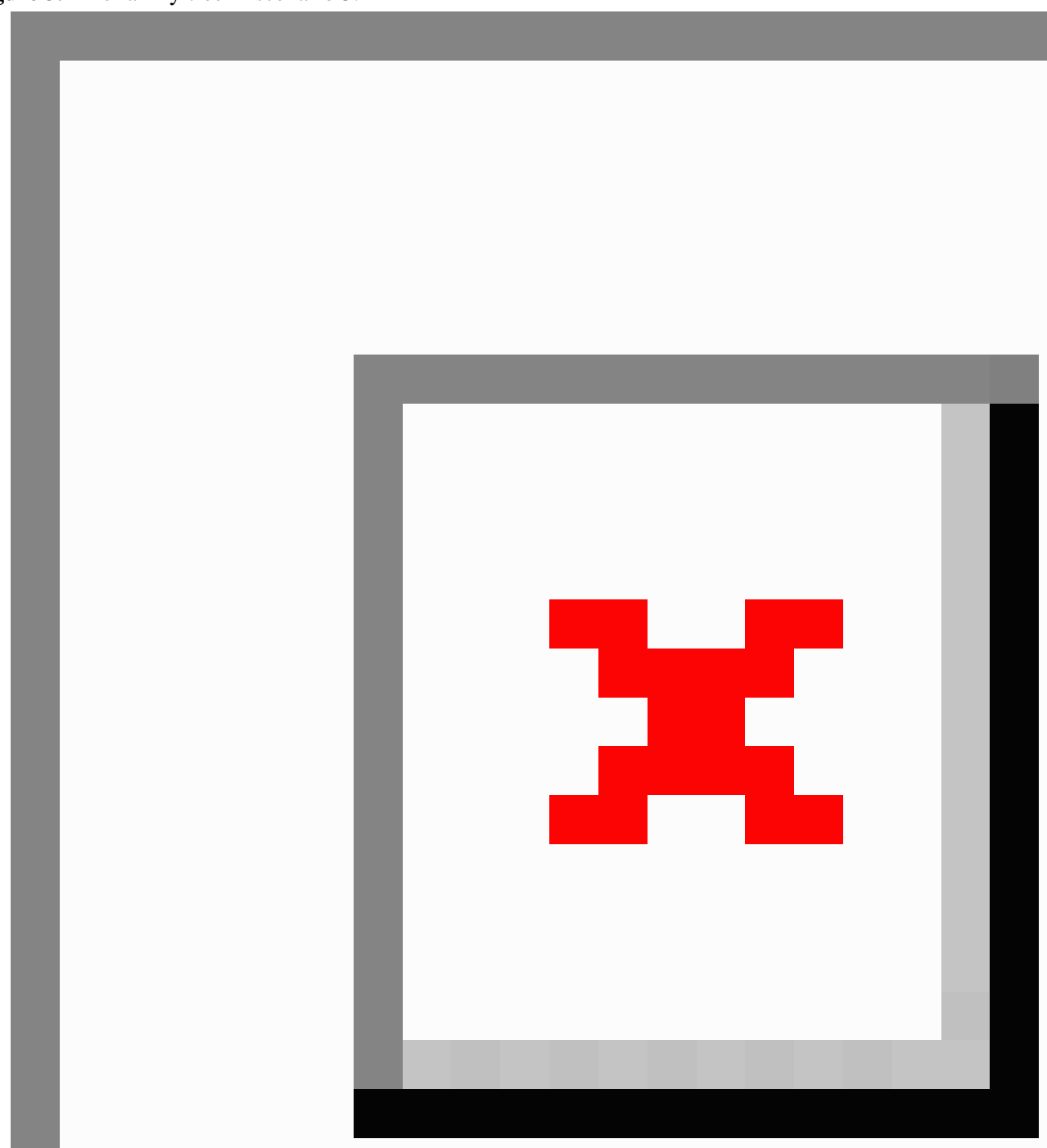

\section{Results}

We developed a system that applied the chatbot to the LINE interface (Figure 4). We then created a conversation with the chatbot according to the 3 devised scenarios. The interface is shown in Figure 5, and the contents of the conversation are shown in Multimedia Appendices 1-3. Figures 6 and 7 show examples of the results obtained during a conversation with the chatbot. Scenarios 1 and 2 met the criteria, whereas scenario 3 did not. This result agreed with the assessments of the 3 experts.
Specifically, the comparisons of the family histories detected by the chatbot with the actual scenarios indicated that each result was consistent with each scenario. The times required for the conversations were 7 minutes for scenario 1, 15 minutes for scenario 2, and 16 minutes for scenario 3. The 3 experts retrospectively reviewed the conversations with the chatbot and noted no errors from the perspective of genetic medicine.

The 3 experts also confirmed the accuracy of the presented family histories and evaluated the accuracy of the presented final test results using the chatbot system. 
Figure 4. The algorithm of the study.

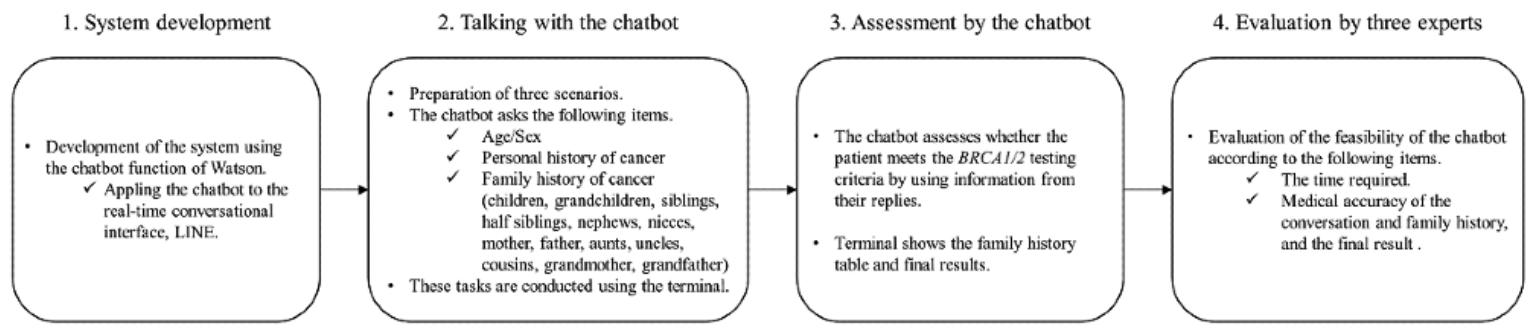

Figure 5. The interface of the chatbot. The LINE interface was used. The conversation in Japanese was translated into English.
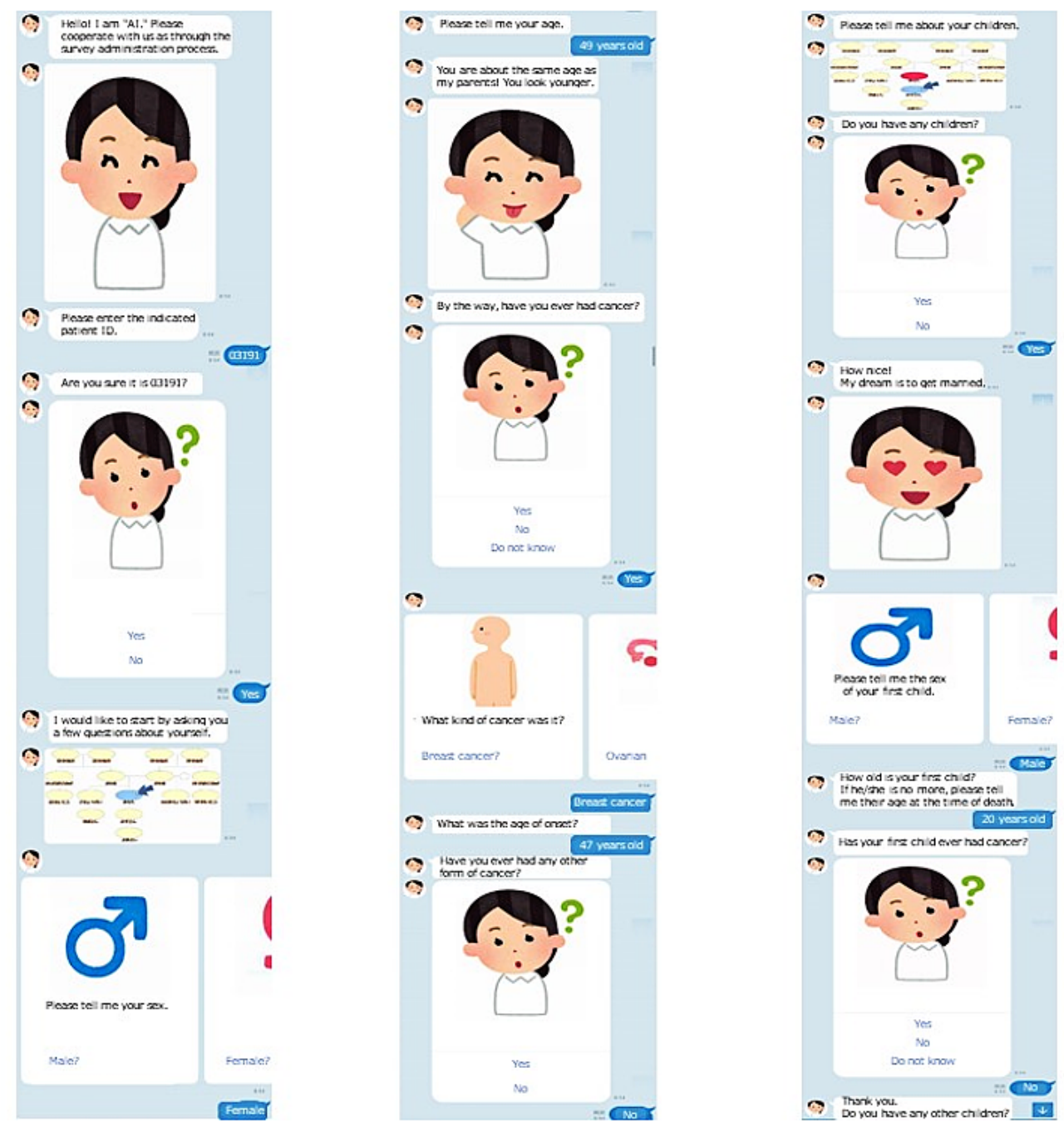
Figure 6. Snapshot of the results of a preliminary screening by the chatbot for scenario 1, according to the criteria of the National Comprehensive Cancer Network (NCCN). We have partially modified the criteria according to our clinical practice at the Kanagawa Cancer Center. The results were originally presented in Japanese and translated into English. Black font: unmet items; red font: met items; gray font: items that had not been asked by this system; yellow square: the final result; "close blood relative:" includes first-, second-, and third-degree relatives.

Recommendation of genetic testing based on BRCA1/2 testing criteria

Meeting one or more of these criteria warrants further personalized risk assessment, genetic counseling, and often genetic testing and management.

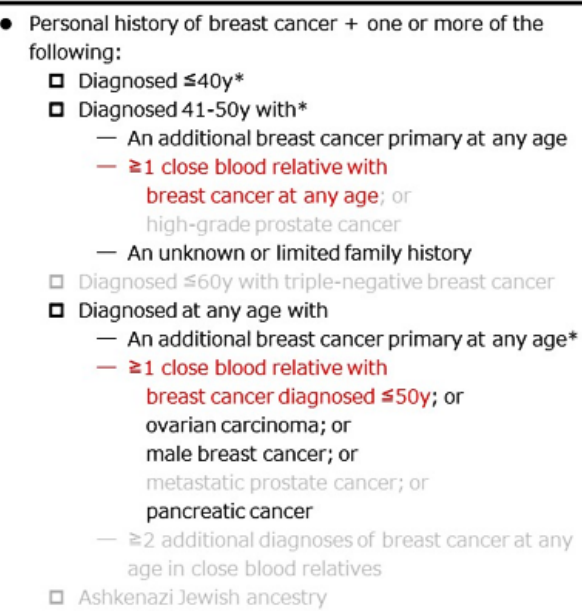

- Personal history of breast cancer + one or more of the following:

ㅁ Diagnosed $\leq 40 y^{*}$

口 Diagnosed 41-50y with*

- An additional breast cancer primary at any age

$-\geq 1$ close blood relative with

breast cancer at any age;

- An unknown or limited family history

ㅁ Diagnosed $\leq 60 \mathrm{y}$ with triple

- An additional breast cancer primary at any age*

$-\geq 1$ close blood relative with

breast cancer diagnosed $\leq 50 \mathrm{y}$; or

ovarian carcinoma; or

male breast cancer; or

pancreatic cancer

pancreatic cancer

noses of breast cancer at any

age in close blood relatives

ㅁ Ashkenazi lewish ancestry

- $1 \geq$ personal history of the following:

口 Personal history of ovarian carcinoma

口 Personal history of male breast cancer

口 Personal history of pancreatic cancer

I. Personal history of metastatic proctate cance

$\square$ Personal history of high-grade prostate cancer at any age witt

- $\geq 1$ close blood relatives with ovarian carcinoma.

pancreatic cancer, or metastatic prostate cancer at any

age or breast cancer <50y; or

- $\geq 2$ close blood relatives with breast, or prostate

cancer(any grade) at any age

An individual not meeting the other criteria but with $\geq 1$ first- or second-degree blood relatives meeting any of the above criteria.

Result

[Meet BRCA1/2 testing criteria]

However, the following items have not been asked.

- Triple negative breast cancer

- Breast cancer with multiple lesions

- Metastatic prostate cancer

- Ashkenazi Jewish ancestry

- Partial modification from NCCN Guidelines (Version3.2019) 
Figure 7. Snapshot of the family history obtained as a result of the preliminary screening by the chatbot for scenario 1 . The results were originally presented in Japanese and translated into English. The screen of the terminal presents one of the results. F: female; M: male: y: years of age.

\begin{tabular}{|c|c|c|c|c|}
\hline \multirow[t]{2}{*}{ Relationship } & \multirow[t]{2}{*}{ Age } & \multirow[t]{2}{*}{ Sex } & \multicolumn{2}{|c|}{ Medical history } \\
\hline & & & \begin{tabular}{|c|} 
Onset \\
age
\end{tabular} & $\begin{array}{l}\text { Cancer } \\
\text { type }\end{array}$ \\
\hline $\begin{array}{l}\text { The person } \\
\text { in question }\end{array}$ & $49 y$ & $\mathrm{~F}$ & $47 y$ & $\begin{array}{l}\text { Breast } \\
\text { cancer }\end{array}$ \\
\hline Child & $20 y$ & M & & \\
\hline Child & $17 y$ & $\mathrm{~F}$ & & \\
\hline \multicolumn{3}{|l|}{ Grandchild } & & \\
\hline Sibling & $53 y$ & $M$ & & \\
\hline Sibling & $46 y$ & $\mathrm{~F}$ & $37 y$ & $\begin{array}{l}\text { Breast } \\
\text { cancer }\end{array}$ \\
\hline \multicolumn{3}{|l|}{ Nephew/Niece } & & \multirow[b]{5}{*}{$\begin{array}{l}\text { Other } \\
\text { cancer }\end{array}$} \\
\hline \multicolumn{3}{|l|}{ Half sibling } & & \\
\hline Father & $81 y$ & M & & \\
\hline $\begin{array}{l}\text { Paternal } \\
\text { uncle/aunt }\end{array}$ & $60 s$ & $M$ & & \\
\hline $\begin{array}{l}\text { Paternal } \\
\text { uncle/aunt }\end{array}$ & $75 y$ & $\mathrm{~F}$ & $70 \mathrm{~s}$ & \\
\hline $\begin{array}{l}\text { Paternal } \\
\text { cousin }\end{array}$ & $50 \mathrm{~s}$ & $\mathrm{~F}$ & \multirow{2}{*}{\begin{tabular}{|l|}
\multicolumn{2}{|l|}{} \\
$60 \mathrm{~s}$ \\
\end{tabular}} & \multirow{2}{*}{$\begin{array}{l} \\
\text { Other } \\
\text { cancer }\end{array}$} \\
\hline $\begin{array}{l}\text { Paternal } \\
\text { grandfather }\end{array}$ & $70 \mathrm{~s}$ & $M$ & & \\
\hline $\begin{array}{l}\text { Paternal } \\
\text { grandmother }\end{array}$ & $80 \mathrm{~s}$ & $\mathrm{~F}$ & & \multirow{3}{*}{$\begin{array}{l}\text { Breast } \\
\text { cancer } \\
\end{array}$} \\
\hline Mother & $78 y$ & $\mathrm{~F}$ & & \\
\hline $\begin{array}{l}\text { Maternal } \\
\text { uncle/aunt }\end{array}$ & $67 y$ & $\mathrm{~F}$ & $49 \mathrm{y}$ & \\
\hline $\begin{array}{l}\text { Maternal } \\
\text { cousin }\end{array}$ & $40 \mathrm{~s}$ & $M$ & & \\
\hline $\begin{array}{l}\text { Maternal } \\
\text { grandfather }\end{array}$ & $60 s$ & $M$ & & \\
\hline $\begin{array}{l}\text { Maternal } \\
\text { grandmother }\end{array}$ & $50 \mathrm{~s}$ & $\mathrm{~F}$ & & \\
\hline
\end{tabular}

\section{Discussion}

\section{Principal Findings}

This study demonstrated that the chatbot system could be applied to preliminary screening for $\mathrm{HBOC}$ in a genetic medicine setting. Our developed system allowed us to achieve the following points required for clinical application: First, our system automatically asked for information items about family history that addressed most of the NCCN guidelines. Thus, the preliminary screening can be performed by a chatbot rather than by a CGC. It cannot completely substitute for a CGC; however, using a chatbot for simpler tasks such as a primary screening would allow a CGC to focus on more complicated clinical practices that require more effort. Second, it was possible to ensure medical accuracy; although the development of the system received technical support from information technology 
companies, the design was created by health care professionals. Accordingly, health care professionals can also change and modify the contents of the conversations regarding the clinical application and continue developing the system while ensuring medical accuracy. Third, the number of correspondences could theoretically be increased to an infinite degree, and therefore, the scale could also be increased. Consequently, the numbers of identified high-risk patients and hospitals performing preliminary screenings could be increased. This offers great promise for the field of genetic medicine.

In this study, we communicated with the chatbot by description-type responses and selection-type responses. This approach was adopted to obtain regular information from existing chatbots $[14,15]$. As a result, the response time could be reduced relative to the time required to provide only descriptive responses. Furthermore, although selection-type responses are frequently used in chatbots that are commercialized for customer services, our preliminary screening scenario was different. Here, the patient did not voluntarily access the chatbot for the purpose of inquiry but was asked to engage with the system by health care professionals. Therefore, it was necessary to devise an order of questions that would enable an easy recall of the family history and develop a persona that would enable the patient to complete the answers without getting tired. However, previous studies have not emphasized the application of the persona setting in chatbots in the medical field [10-12].

When collecting a family history using our newly developed chatbot, we assumed that AI and the patient would discuss family. Therefore, we created detailed family information for AI and included this in her persona. To improve the response rate, the development of more useful and effective personae is warranted in future studies. It would also be useful to personalize the chat, which is based on the persona of AI, by selecting from among several personae according to the patient's age. Moreover, chatting based on the persona of AI may or may not be considered excessive, depending on the patient's background.

\section{Limitations}

Several issues should be addressed to ensure the practical application of this system in terms of efficiency and convenience. First, the system must be easy to operate by the patient. A user-friendly interface is desirable. Therefore, we developed a chatbot system using the LINE interface. As noted, LINE is the most popular SNS in Japan, and we assumed that it would be familiar to the patients [13]. However, older adults who are not used to smartphones may need a friendlier interface or human assistance. In Japan, the personal ownership rate and use of smartphones have been increasing since 2010. Remarkably, the associated generational gap is large, as only $18.8 \%$ and $6.1 \%$ of those in their $70 \mathrm{~s}$ and $80 \mathrm{~s}$ have reported owning such phones, respectively, compared to more than $90 \%$ of those in their 20s and 30s [16]. Therefore, the development of a system that considers both age and information technology literacy would be required. Although the supporting personnel would not necessarily need to be CGCs, sufficient personnel would be required to support multiple patients in parallel. Moreover, both elderly and visually impaired patients may find it difficult to operate a smartphone without voice assistance. In this case, it may be difficult to determine the significance of using the AI screening system.

Second, the patient must complete all queries during the screening process. In addition to the persona setting, a device that does not bore the patient is required. A change in the depth of the information heard during preliminary screening would also be necessary. We identified high-risk patients in our institution by interviewing all patients with breast cancer who visited the outpatient department (preliminary screening). As there is a limited amount of time before the medical examination, the information reported by the patient is often limited to relatives who have had cancer rather than the family structure (grandparents, parents, uncles and aunts, cousins, siblings, nephews and nieces, and children) and medical histories. Although our new system allows us to listen to each person's medical history after determining the family structure, it may be useful to adjust the system further depending on the purpose (eg, focusing only on family members suffering from cancer).

Third, we must consider how to handle personal information. We developed a system that is interacted with using a terminal (ie, a smartphone or tablet). However, the secure retention of data requires further exploration. The linking of the obtained results with electronic medical records would be an appropriate handling method.

Finally, clinical trials using this system are required; here, the device would be returned to the health care professionals once the patient has finished the conversation. In this study, the experts responded to the items based on the created scenarios. Evaluation by 3 predefined scenarios is limited system validation. Thus, we have conducted a study with a small number of patients. We would expect that the time required to input answers for actual patients would be longer because they would also need to recall the family history. In addition, human error (such as incorrectly entering a patient ID or confusing the appropriate terminal with another patient's terminal) may occur when patients being screened are also simultaneously treated at the outpatient department. Measures to prevent such errors may also be required. Therefore, it is first necessary to conduct further studies to solve any problem with this system on a small number of people before further investigation is engaged.

\section{Conclusions}

We demonstrated that the chatbot system could be applied to preliminary screening for HBOC in a genetic medicine setting. Our system could automatically ask for family history items that covered most of the NCCN guidelines without requiring an actual person and remained automated up to the screening result. Health care professionals determined the system design; thus, it was possible to ensure medical accuracy. Theoretically, the number of correspondences and interactions could be increased to an infinite degree, and therefore, the scale could also be increased. The system may also apply to other diseases for which the screening criteria are based on family history. For future clinical applications, it will be necessary to conduct clinical research and to further improve the efficiency and convenience of the system. 


\section{Acknowledgments}

We thank Advanced Integration Technology (AIT) Inc for providing technical support during the development of the chatbot system. We also thank Editage for English language editing. This study was supported by the Kanagawa Prefectural Hospitals Cancer Fund.

\section{Conflicts of Interest}

Hiroto Narimatsu received a research fund from Chugai Pharmaceutical Co., Ltd. The other authors have no conflicts of interest to declare.

\section{Multimedia Appendix 1}

Summary and chat contents of scenario 1 .

[DOCX File, 23 KB-Multimedia Appendix 1]

\section{Multimedia Appendix 2}

Summary and chat contents of scenario 2 .

[DOCX File, 22 KB-Multimedia Appendix 2]

\section{Multimedia Appendix 3}

Summary and chat contents of scenario 3 .

[DOCX File, $30 \mathrm{~KB}$-Multimedia Appendix 3]

\section{References}

1. Latest Cancer Statistics. National Cancer Center for Cancer Control Information Services. URL: https://ganjoho.jp/reg_stat/ statistics/stat/summary.html [accessed 2019-04-24]

2. Schwartz GF, Hughes KS, Lynch HT, Fabian CJ, Fentiman IS, Robson ME, Consensus Conference Committee The International Consensus Conference Committee. Cancer 2008 Nov 15;113(10):2627-2637 [FREE Full text] [doi: 10.1002/cncr.23903] [Medline: 18853415]

3. Turnbull C, Rahman N. Genetic predisposition to breast cancer: past, present, and future. Annu Rev Genomics Hum Genet 2008;9:321-345. [doi: 10.1146/annurev.genom.9.081307.164339] [Medline: 18544032]

4. Tung N, Lin NU, Kidd J, Allen BA, Singh N, Wenstrup RJ, et al. Frequency of Germline Mutations in 25 Cancer Susceptibility Genes in a Sequential Series of Patients With Breast Cancer. J Clin Oncol 2016 May 01;34(13):1460-1468 [FREE Full text] [doi: 10.1200/JCO.2015.65.0747] [Medline: 26976419]

5. Momozawa Y, Iwasaki Y, Parsons MT, Kamatani Y, Takahashi A, Tamura C, et al. Germline pathogenic variants of 11 breast cancer genes in 7,051 Japanese patients and 11,241 controls. Nat Commun 2018 Oct 04;9(1):4083 [FREE Full text] [doi: 10.1038/s41467-018-06581-8] [Medline: 30287823]

6. Kuchenbaecker KB, Hopper JL, Barnes DR, Phillips K, Mooij TM, Roos-Blom M, BRCA1BRCA2 Cohort Consortium, et al. Risks of Breast, Ovarian, and Contralateral Breast Cancer for BRCA1 and BRCA2 Mutation Carriers. JAMA 2017 Jun 20;317(23):2402-2416. [doi: 10.1001/jama.2017.7112] [Medline: 28632866]

7. NCCN Guidelines. National Comprehensive Cancer Network (NCCN). 2019. URL: https://www.nccn.org/professionals/ physician gls/pdf/genetics screening.pdf [accessed 2019-04-24]

8. Childers CP, Childers KK, Maggard-Gibbons M, Macinko J. National Estimates of Genetic Testing in Women With a History of Breast or Ovarian Cancer. J Clin Oncol 2017 Dec 01;35(34):3800-3806 [FREE Full text] [doi: 10.1200/JCO.2017.73.6314] [Medline: 28820644]

9. Certified GCIC. Certified Genetic Counselor Institutional Committee. Genetic Society of Japan. URL: http://plaza.umin.ac.jp/ $\sim \mathrm{GC} /$ [accessed 2019-04-24]

10. Chaix B, Bibault J, Pienkowski A, Delamon G, Guillemassé A, Nectoux P, et al. When Chatbots Meet Patients: One-Year Prospective Study of Conversations Between Patients With Breast Cancer and a Chatbot. JMIR Cancer 2019 May 02;5(1):e12856 [FREE Full text] [doi: 10.2196/12856] [Medline: 31045505]

11. Greer S, Ramo D, Chang Y, Fu M, Moskowitz J, Haritatos J. Use of the Chatbot "Vivibot" to Deliver Positive Psychology Skills and Promote Well-Being Among Young People After Cancer Treatment: Randomized Controlled Feasibility Trial. JMIR Mhealth Uhealth 2019 Oct 31;7(10):e15018 [FREE Full text] [doi: 10.2196/15018] [Medline: $\underline{31674920}$ ]

12. Piau A, Crissey R, Brechemier D, Balardy L, Nourhashemi F. A smartphone Chatbot application to optimize monitoring of older patients with cancer. Int J Med Inform 2019 Aug;128:18-23. [doi: 10.1016/j.ijmedinf.2019.05.013] [Medline: 31160007] 
13. Special Feature Sustainable Growth by ICT in the Age of Declining Population: Social media usage status. Ministry of Internal Affairs and Communications. 2018. URL: http://www.soumu.go.jp/johotsusintokei/whitepaper/ja/h30/html/ nd142210.html [accessed 2019-04-24]

14. mineo user support page. OPTAGE Inc. 2019. URL: https://support.mineo.jp/mai/chat/ [accessed 2019-04-19]

15. Rakuten Ichiba Inquiry Chat. Rakuten Inc. 2019. URL: https://chat.ichiba.faq.rakuten.co.jp/ [accessed 2019-04-19]

16. Special Feature Sustainable Growth by ICT in the Age of Declining Population: Expansion of internet use. Ministry of Internal Affairs and Communications. 2018. URL: http://www.soumu.go.jp/johotsusintokei/whitepaper/ja/h30/html/ nd142110.html [accessed 2019-04-24]

\section{Abbreviations \\ AI: augmented intelligence \\ BRCA1/2: BRCA1 or BRCA2 \\ CGC: certified genetic counselor \\ HBOC: hereditary breast and ovarian cancer \\ NCCN: National Comprehensive Cancer Network \\ SNS: social network service}

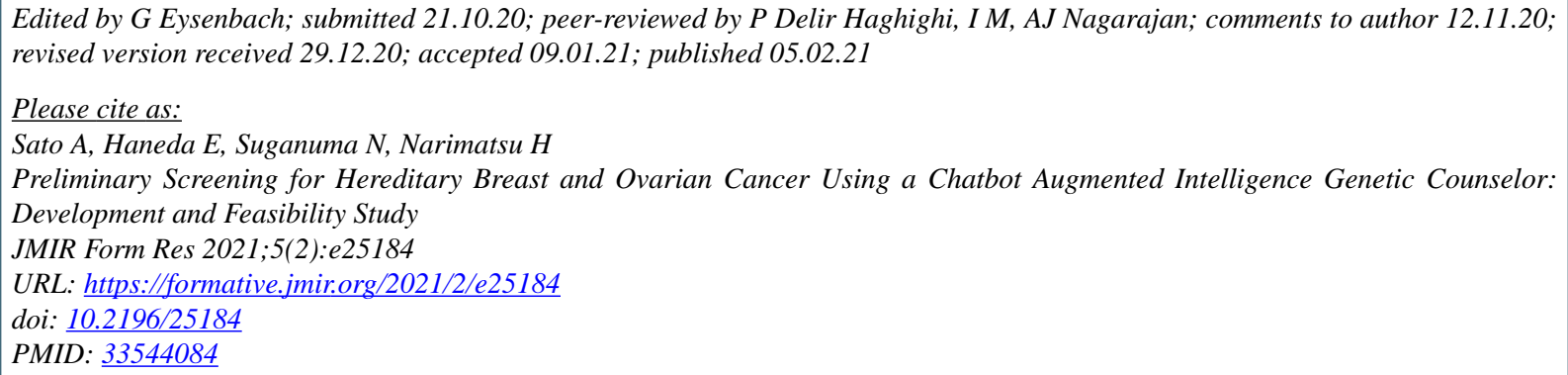

(C)Ann Sato, Eri Haneda, Nobuyasu Suganuma, Hiroto Narimatsu. Originally published in JMIR Formative Research (http://formative.jmir.org), 05.02.2021. This is an open-access article distributed under the terms of the Creative Commons Attribution License (https://creativecommons.org/licenses/by/4.0/), which permits unrestricted use, distribution, and reproduction in any medium, provided the original work, first published in JMIR Formative Research, is properly cited. The complete bibliographic information, a link to the original publication on http://formative.jmir.org, as well as this copyright and license information must be included. 